INFECTION CONTROL AND HOSPITAL EPIDEMIOLOgY MAY 2007, VOL. 28, NO. 5

\title{
Assessment of Intervention Measures for the 2003 SARS Epidemic in Taiwan by Use of a Back-Projection Method
}

\author{
Paul S. F. Yip, PhD; Y. H. Hsieh, PhD; Ying Xu, MPhil; K. F. Lam, PhD; C. C. King, PhD; H. L. Chang, MD
}

\begin{abstract}
oвjectives. To reconstruct the infection curve for the 2003 severe acute respiratory syndrome (SARS) epidemic in Taiwan and to ascertain the temporal changes in the daily number of infections that occurred during the course of the outbreak.

METHOD. Back-projection method.

RESUlts. The peaks of the epidemic correspond well with the occurrence of major infection clusters in the hospitals. The overall downward trend of the infection curve after early May corresponds well to the date (May 10) when changes in the review and classification procedure were implemented by the SARS Prevention and Extrication Committee.
\end{abstract}

CONCLUSION. The major infection control measures taken by the Taiwanese government over the course of the SARS epidemic, particularly those regarding infection control in hospitals, played a crucial role in containing the outbreak.

Infect Control Hosp Epidemiol 2007; 28:525-530

The severe acute respiratory syndrome (SARS) epidemic of 2003 presented one of the most serious global health threats since the emergence of human immunodeficiency virus (HIV). Uncertainty about clinical and epidemiological aspects of the novel SARS coronavirus made it difficult to determine appropriate public health interventions to contain the epidemic during the course of the outbreak. ${ }^{1-3}$ Despite the lack of a quantitative method to justify the effectiveness of intervention measures at the population level, monitoring changes in the daily number of infections over the course of the epidemic may provide some insight into the immediate impact and effectiveness of these measures. An infection curve is a plot of the daily counts of new infections over the course of the epidemic. The infection curve for an epidemic would be a useful and convenient tool for evaluating the effectiveness of intervention measures. ${ }^{4}$ However, the daily number of infections is unobservable. The available information on the daily number of reported cases, with an unknown individual disease incubation period, is not sensitive enough to reflect the spread of the epidemic over the course of the outbreak. The immediate effect of the intervention measures is therefore difficult to ascertain. Nevertheless, prompt and decisive action is needed in efforts to contain newly emerging epidemics, such as the potential threat of pandemic influenza. Hence, a procedure for the construction of an infection curve using statistical methods is highly desirable.
In this study, we adopted a back-projection method similar to that used by Chau and Yip ${ }^{4}$ to examine the SARS epidemic in Taiwan. The back-projection method has been widely used in modeling the spread of HIV infection and acquired immune deficiency syndrome..$^{5-7}$ The clinical course of SARS progresses from infection, through incubation, to onset of symptoms, so the back-projection technique can be readily used in this context. With updated SARS case data, we were able to reconstruct the infection curve for the SARS outbreak in Taiwan and show that the fluctuations on the infection curve fit well with the major events on the time line of that outbreak. A discussion of the public health implications of our results is also provided here.

\section{METHODS}

Data

According to the Taiwan Center for Disease Control (TCDC) ${ }^{8}$ and the World Health Organization (WHO), ${ }^{9} 346$ patients in Taiwan were officially confirmed as having SARS. Among them, there were 37 deaths directly caused by SARS (ie, the cause of death was recorded as SARS) and 36 SARS-related deaths (ie, the cause of death was not directly attributed to SARS). However, in a follow-up study done in collaboration with the TCDC to track previously unconfirmed cases, a total of 134 additional SARS coronavirus antibody-positive pa-

From the Department of Statistics and Actuarial Science, University of Hong Kong, Hong Kong (P.S.F.Y., Y.X., K.F.L.); and the Department of Applied Mathematics, National Chung Hsing University, Taichung (Y.H.H.), the Institute of Epidemiology, College of Public Health, National Taiwan University (C.C.K.), and the Center for Disease Control, Department of Health (H.L.C.), Taipei, Taiwan

Received June 21, 2006; accepted August 24, 2006; electronically published March 30, 2007.

(C) 2007 by The Society for Healthcare Epidemiology of America. All rights reserved. 0899-823X/2007/2805-0003\$15.00. DOI: 10.1086/516656 
tients were found to have laboratory-confirmed cases of SARS, 12 of whom died. ${ }^{10}$ Consequently, the total number of confirmed SARS cases in Taiwan by the end of December 2004 was 480, which is different from the figure cited in earlier published reports on SARS in Taiwan. ${ }^{11-15}$ The definition of a SARS case used in Taiwan is the same as that used by the WHO. ${ }^{14,16}$ These 480 cases with onset of symptoms occurring between February 25, 2003, and June 25, 2003, were used in the present study. To our knowledge, this is the first backprojection study of the Taiwan SARS outbreak that makes use of the more comprehensive data set of laboratory-confirmed SARS cases.

\section{Statistical Analysis}

We proposed to use the back-projection method to estimate the daily counts of newly infected people, or the mean daily number of new infections. The back-projection method assumes a known distribution for the incubation period on the basis of data from other studies. The details of the model employed, including the model assumptions and model parameters, are given in Appendix A. For our study, we assumed that the incubation period followed a gamma distribution. The details pertaining to the choice of gamma distribution can be found in Appendix B.

It has been shown that age was an important factor in the SARS case-fatality rate. ${ }^{20}$ Similarly, it was of interest here to examine how age was related to infection intensity. The estimates of the daily number of infections would certainly be more accurate and precise, given the additional information, if age was an important factor. ${ }^{5}$ The Kruskal-Wallis test was employed to test for the homogeneity of incubation distributions among 3 age groups: 0-14 years, $15-59$ years, and 60 years and older. The effect of age was not significant; the null hypothesis of homogeneity was not rejected at the $5 \%$ significance level $(P=.36)$. Therefore, no age-adjusted expectation-maximization-smoothing algorithm ${ }^{4}$ (EMS; see Appendix B) was used to estimate the infection curve of the Taiwan SARS epidemic.

\section{RESULTS}

Figure 1 gives the estimated daily number of SARS infections, $\lambda_{t}$, from February 18 to June 17, 2003. The point-wise 95\% confidence interval for $\lambda_{t}$ was constructed for each time point $t$ by using a bootstrapping method. ${ }^{6}$ A chronological summary of intervention measures taken in Taiwan ${ }^{21}$ is also given in Figure 1.

The major peaks of the estimated $\lambda_{t}$ values at the height of the outbreak in Taiwan corresponded well with the occurrences of major SARS-related events in Taiwan hospitals. The first peak, identified as occurring on approximately April 17, corresponded to the infection clusters at Hoping Hospital and Jenchi Hospital that resulted in the escalation of the outbreak in Taiwan and, subsequently, the shutdown of Hoping Hospital on April 24 and the shutdown of Jenchi Hos- pital 2 days later. The second peak, identified as occurring on approximately April 25, corresponded to the infection clusters at the National Taiwan University Hospital emergency department (which was shut down on May 12), McKay Hospital, and Chung-Hsing Hospital in Taipei. ${ }^{14,22}$ The third and last peak, identified as occurring on approximately May 5 , corresponded to the nosocomial infection cluster at Kaohsiung Chang Gung Memorial Hospital in the southern city of Kaohsiung. A smaller peak occurring on approximately May 15 corresponded to the infection cluster at Kuandu Hospital in Taipei.

During the height of the outbreak (from mid-April to early May), the trough in estimated $\lambda_{t}$ values occurring on approximately April 21 corresponded to an infection cluster at Hoping Hospital being reported to the health authorities on April 21 and 22, resulting in a heightened alertness for hospital infection control personnel that lessened the spread of nosocomial infections at Hoping Hospital. The second trough, occurring on approximately April 30, corresponded well to the implementation of level B quarantine policy on April 28; this marked the start of large-scale border control and home quarantine, which turned out to be the major turning point for ending the outbreak in Taiwan. ${ }^{23}$ Furthermore, the overall downward trend of the infection curve after early May corresponded well to a change in review and classification procedures implemented by the cabinet-level SARS Prevention and Extrication Committee in Taiwan on May 10 to expedite the review and reclassification of suspected SARS cases in an effort to quickly identify and isolate the patients who truly had SARS. ${ }^{21}$ April 28 was determined to be an important date for significantly expediting quick identification of suspected cases (thereby decreasing the time between onset and diagnosis), and May 10 was an important date for swiftly classifying probable cases (thereby decreasing the time between diagnosis and reclassification). ${ }^{10}$

\section{I S C US S I O N}

The estimated daily number of infections during the course of an epidemic is a good indicator of the infection intensity. The immediate benefit of certain effective intervention measures is easily seen because of their influence on reducing the daily number of infections. With the convolution of the incubation period, the time at which a patient is infected is unobservable. Hence, it is desirable to use statistical methods to reconstruct the infection curve that allow for the evaluation of the effectiveness of intervention measures. The back-projection method, which is a very useful tool in modeling epidemics, can be readily used in this context.

The use of the back-projection method, relying on the aggregate observed daily number of patients with confirmed SARS, to construct the infection curve has advantages over tracing the patients' contact history, as done by Karlberg et al. $^{24}$ The case-study method ${ }^{24}$ is very useful in understanding the epidemic. However, it is not efficient and may be very 


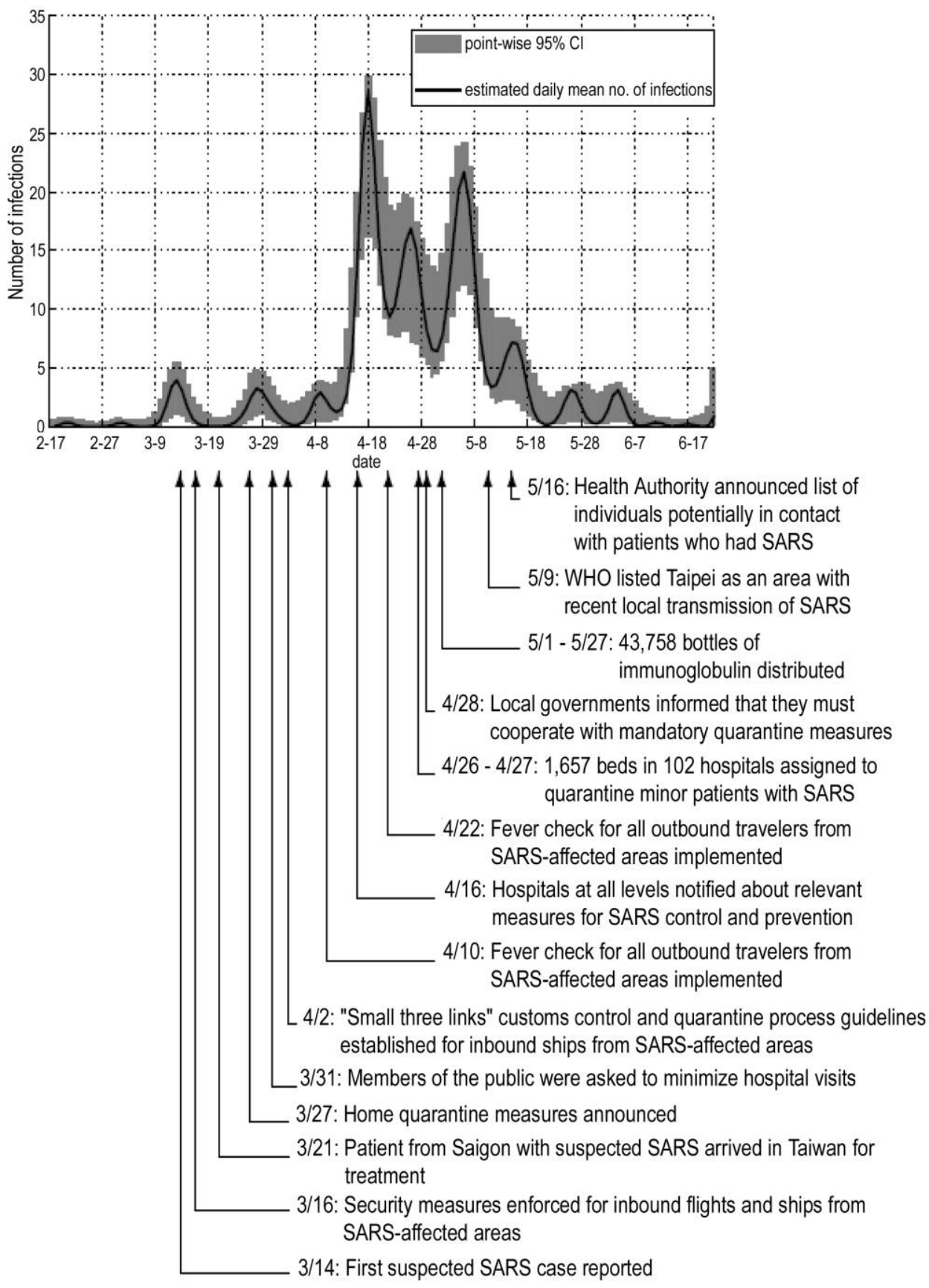

FIGURE. Time line showing estimated daily mean number of severe acute respiratory syndrome (SARS) cases (and point-wise 95\% confidence intervals [CIs]) during the 2003 outbreak in Taiwan and major infection control interventions. WHO, World Health Organization. ${ }^{21}$

time consuming, because a detailed contact history for each individual is needed. Moreover, such information is usually unavailable and/or unreliable, especially during the course of the epidemic, and the infection curve estimated by the casestudy method is subject to individual fluctuations. The estimated infection curve obtained from the back-projection method smooths out these fluctuations so that waves of in- fection can be clearly identified. Chau and Yip ${ }^{4}$ used the backprojection method to analyze the 2003 SARS outbreak in Hong Kong. They managed to show that 4 waves of infection had occurred over the course of that outbreak. It is interesting to note that 3 of the 4 waves (ie, all except the outbreak at Amoy Garden $)^{4}$ originated in hospitals.

Other similar studies on the evaluation of intervention 
measures used during SARS outbreaks can be found in Hsieh et al. ${ }^{23}$ and Pang et al. ${ }^{25} \mathrm{Hsieh}$ et al. ${ }^{23}$ applied the Richards model $^{26}$ to the daily cumulative number of cases during the Taiwan SARS outbreak, and they estimated that April 28 was the turning point of the outbreak: on this date, the growth rate of the cumulative number of cases attained its maximum level, decreasing gradually thereafter. However, this method does not provide information on each major and minor wave of the epidemic. Pang et al. ${ }^{25}$ utilized the time lag between onset of illness and hospitalization during the Beijing SARS epidemic. This method is very simple and can be computed quite easily by hand. However, the evaluation of control measures with this method was further complicated by the time lag of at least 1 incubation period between implementation of the measure and the time that the intervention took effect.

The Taiwan SARS outbreak displayed a pattern very similar to that of the Hong Kong outbreak. Each major peak of the outbreak corresponds to infection clusters in hospitals. If the infection control measures in affected hospitals were more effective, it is likely that the epidemic could be contained within the hospital compounds and would not be able to spread as readily to other patients, hospital staff, and visitors in the hospitals, and, subsequently, would not spread as readily to the community. There are a number of issues that need to be critically addressed in the control and prevention of SARS or any emerging infectious disease in hospitals. The availability of isolated wards to prevent cross-infection between patients with and patients without SARS, as well as the adequate supply of protective equipment and medical items, all play important parts in containing the epidemic. Nevertheless, the professionalism exhibited by the medical and healthcare staff during the outbreak, in terms of their dedication, sacrifice, and medical knowledge and training, is also a crucial factor in combating the disease.

Although infection intensity did not show significant age dependence, the SARS fatality rate was found to be age dependent. ${ }^{20}$ It is also important to advise people to seek appropriate medical assistance immediately after the onset of possible symptoms. However, hospitalization might actually increase the risk of infection as a result of an insufficient number of isolation wards in hospitals and overstressed medical staff dealing with an influx of persons with suspected (but not genuine) cases. Consequently, in the case of a newly emerging infectious disease epidemic, quick identification and isolation of patients with actual and highly probable cases in a single ward in a hospital is essential to control the epidemic and to prevent the spread of infection in the hospital. To achieve this, some effective discriminatory authority during the course of the epidemic is very important. ${ }^{27,28}$

The Taiwanese government implemented numerous intervention measures, including 2 levels of quarantine. Level A quarantine applied to people who potentially had contact with infectious individuals, and level B quarantine applied to travelers coming from affected areas abroad. More than 150,000 people were notified and placed under home quarantine, us- ing limited contact tracing and border control measures. Only 24 of these quarantined individuals turned out to have SARS,${ }^{10}$ which accounted for only $5 \%$ of all confirmed SARS cases. One should note, however, that full-scale (ie, level A and level B) quarantine in Taiwan was not implemented until April 28, when several major hospital-based infection clusters had already occurred. ${ }^{27}$ Therefore, swift home quarantine of potentially exposed individuals and voluntary quarantine of individuals with subclinical symptoms should be considered. These could be effective complementary intervention measures to prevent people who are potentially infectious from spreading the epidemic further in the community. In addition, these measures could prevent hospitals from being overburdened with patients who have suspected cases that turn out not to be genuine. Such complementary measures are particularly relevant if no effective medical treatment is readily available during the early stages of emerging novel infectious diseases, as in the case of SARS in 2003 or the potential mutation of avian influenza virus (H5N1) to a strain that can be transmitted from person to person.

\section{ACKNOWLEDGMENTS}

We thank the referees for their constructive comments. The work was initiated when P.S.F.Y., Y.H.H., and Eric H.Y. Lau, PhD, met in an infectious disease modeling workshop hosted by the Institute of Mathematics and Statistics of the National University of Singapore in September 2005.

Financial support. This research was supported by a Research Grants Council of the Hong Kong Special Administrative Region grant (to P.S.F.Y) and a National Science Council (NSC-Taiwan) research grant (NSC-94-2314B-005-002; to Y.H.H).

Potential conflicts of interest. All authors report no conflicts of interest relevant to this article.

\section{A P PENDIX A}

\section{The Model}

Let $t=1,2, \ldots, T$ be the time units (in days) for the data. Note that the first recognized SARS patient in Taiwan was a 54-year-old businessperson who traveled to Guangdong Province, China, on February 5, 2003, returned to Taipei via Hong Kong on February 21, and had onset of symptoms on February 25 . Given that most estimates of the SARS incubation period are less than 8 days, ${ }^{16-18}$ it is therefore unlikely that the infection would have occurred before February 18, if we take into account of the length of the incubation period. Therefore, February 18 is assumed to be the start of the epidemic in Taiwan, and this date is denoted $t=1$. The latest data available for analysis were from June 25, 2003, and thus $T$ is set at 128 days.

\section{Parameters}

The variable $\mu_{t}$ indicates the expected number of individuals with onset of symptoms on day $t$. The $\lambda_{t}$ variable indicates the expected number of infections on day $t$. The variable $f_{t, d}$ indicates the probability that an individual is infected on 
day $t$ and has an incubation period of $d$ days. The variable $N_{t, d}$ indicates the number of individuals infected on day $t$ with an incubation period of $d$ days. The variable $Y_{t}$ indicates the number of reported and/or confirmed cases on day $t$.

\section{Assumptions}

The daily numbers of infections are assumed to follow independent Poisson processes with intensities $\lambda_{t}$ for $t=$ $1, \ldots, T$. In addition, the daily numbers of confirmed and/ or identified cases are assumed to follow independent Poisson processes with intensities

$$
\mu_{t}=\sum_{s=0}^{t-1} \lambda_{t-s} f_{t-s, s}
$$

for day $t$.

Because the incubation period is relatively short and the basic treatment is quarantine, ${ }^{10}$ the effect of the infection time $d$ on the incubation period is minimal and may be ignored. Hence, $f_{t-s, s}$ is simplified as $f_{s}$.

\section{A P P ENDIX B}

\section{Expectation-Maximization-Smoothing Algorithm (EMS)}

There are 2 adequate fits to SARS-associated data sets, namely the Weibull and gamma distributions. ${ }^{4,18}$ The complete data log-likelihood function for $\lambda_{t}$ is given as follows:

$$
\log L=\sum_{t=1}^{T} \sum_{d=0}^{T-t}\left\{N_{t, d} \log \left(\lambda_{t} f_{d}\right)-\lambda_{t} f_{d}\right\}
$$

where $f_{d}$ is assumed to follow either a gamma or a Weibull distribution, and the cumulative density functions are

$$
F(t)=\frac{1}{\beta^{\alpha} \Gamma(\alpha)} \int_{0}^{t+0.5} x^{\alpha-1} e^{-x / \beta} d x
$$

or

$$
F(t)=1-\exp \left\{-\left[\frac{t+0.5}{\beta}\right]^{\alpha}\right\}
$$

respectively. Unknown parameters $(\alpha, \beta)$ for the 2 distributions mentioned above can be estimated on the basis of the incubation-days of 98 patients, with their infection dates being identified retrospectively.

In practice, we can only observe $Y_{t}$. Mathematically, we have

$$
Y_{t}=\sum_{d=0}^{t-1} N_{t-d, d}
$$

A convenient tool to estimate $\lambda_{t}$ in a situation in which data are missing is the expectation-maximization (EM) algorithm. However, the estimates of $\lambda_{t}$ may be very erratic, and the interpretation is generally made more sensible by smoothing out the estimates. Hence, the expectation-maximizationsmoothing (EMS) algorithm ${ }^{6}$ was adopted in the current analysis, using the algorithm summarized below.

\section{Expectation Step}

The posterior expectation of $N_{t, d}$ given $Y_{1}, \ldots, Y_{T}$ can be shown to be

$$
E\left(N_{t, d} \mid Y_{1}, Y_{2}, \ldots, Y_{T}\right)=Y_{t+d} \frac{\lambda_{t} f_{d}}{\sum_{i=1}^{t+d} \lambda_{i} f_{t+d-i}}
$$

\section{Maximizing Step}

The maximum likelihood estimates of $\lambda_{t}$ can be obtained by the iterative updating equations

$$
\hat{\lambda}_{t}^{[j+1]}=\frac{\tilde{\lambda}_{t}^{[j]}}{\sum_{d=0}^{T-t} f_{d}} \sum_{d=0}^{T-t} \frac{Y_{t+d} f_{d}}{\sum_{i=1}^{t+d} \tilde{\lambda}_{t}^{[j]} f_{t+d-i}}
$$

where

$$
\tilde{\lambda}_{t}^{[j]}
$$

is the estimate of $\lambda_{t}$ at the $j$-th iteration.

\section{Smoothing Step}

As the fluctuation among the observed daily number of SARS confirmed cases is not that erratic, we can simply adopt a 3width window to smooth out the

$$
\hat{\lambda}_{t}^{[j]}
$$

in each iteration in the M-step. The corresponding weights are $w_{0}=w_{2}=0.1$ and $w_{1}=0.8$. Therefore, we have

$$
\tilde{\lambda}_{t}^{[j+1]}=\sum_{i=0}^{2} w_{i} \hat{\lambda}[j+1] t+i-1 .
$$

Hence, the smoothed estimate of $\lambda_{t}$ is given by

$$
\tilde{\lambda}_{t}=\lim _{j \rightarrow \infty} \tilde{\lambda}_{t}^{[j]}
$$

A gamma form for $f_{d}$ yields a value of -403.01 for the log-likelihood function (1), whereas a Weibull form yields -501.23 , indicating that in the case of the Taiwan SARS outbreak, the incubation distribution is better fitted by the gamma distribution. The estimated mean duration $( \pm S D)$ 
of the incubation period for SARS in Taiwan is $6.33 \pm$ 3.22 days. Donnelly et al. ${ }^{19}$ used a gamma distribution for data on the incubation period of SARS in Hong Kong, with an estimated mean duration $( \pm S D)$ of $6.37 \pm 4.09$ days.

Address reprint requests to Paul Yip, PhD, Department of Statistics and Actuarial Science, University of Hong Kong, Pokfulam Road, Hong Kong (sfpyip@hku.hk).

\section{REFERENCES}

1. Cinati J, Morgenstern B, Bauer G, Chandra R, Rabenau H, Doerr HW. Glycyrrhizin, an active component of liquorice roots, and replication of SARS-associated coronavirus. Lancet 2003; 361:2045-2046.

2. Yip SF, Lam KF, Lau HY, Chau PH, Tsang KW, Chao A. A comparison study of real-time fatality rates: severe acute respiratory syndrome in Hong Kong, Singapore, Taiwan, Toronto and Beijing, China. J R Stat Soc Ser A 2005; 168:233-243.

3. Yip SF, Lau HY, Lam KF, Huggins RM. A chain multinomial model for estimating the real-time fatality rate of a disease, with an application to severe acute respiratory syndrome. Am J Epidemiol 2005; 161:700-706.

4. Chau PH, Yip SF. Monitoring the severe acute respiratory syndrome epidemic and assessing effectiveness of interventions in Hong Kong Special Administrative Region. J Epidemiol Community Health 2003; 57:766-769.

5. Becker NG, Marschner IC. A method for estimating the age-specific relative risk of HIV infection from AIDS incidence data. Biometrika 1993; 80:165-178.

6. Becker NG, Watson LF, Carlin JB. A method of non-parametric backprojection and its application to AIDS data. Stat Med 1991; 10:1527-1542.

7. Brookmeyer R, Gail MH. A method for obtaining short-term projections and lower bounds on the size of the AIDS epidemic. J Am Stat Assoc 1988; 83:301-308.

8. World Health Organization. Summary of probable SARS cases with onset of illness from 1 November 2002 to 31 July 2003. September 26, 2003. Available at: http://www.who.int/csr/sars/country/table2003_09_23/en/. Accessed January 6, 2006.

9. World Health Organization. Summary of probable SARS cases with onset of illness from 1 November 2002 to 31 July 2003. April 21, 2004. Available at: http://www.who.int/csr/sars/country/table2004_04_21/en/index.html. Accessed January 6, 2006.

10. Hsieh YH, King CC, Chen WS, et al. Quarantine for SARS, Taiwan. Emerg Infect Dis 2005; 11:278-282.

11. Chin SC, Lin JH, Lai SK, et al. Testing for the virus load of probable SARS cases in Taiwan. In: Su IJ, ed. Memoir of Severe Acute Respiratory Syndrome Control in Taiwan, 2003. Taipei: CDC-Taiwan; 2003:32-38.
12. Wu YC, Hsiung CA, Chen KT, et al. Comparison of clinical and laboratory profiles between coronavirus positive and negative SARS probable cases in Taiwan. In: Su IJ, ed. Memoir of Severe Acute Respiratory Syndrome Control in Taiwan, 2003. Taipei: CDC-Taiwan; 2003:11-26.

13. Wang JT, Sheng WH, Fang CT, et al. Clinical manifestations, laboratory findings, and treatment outcomes of SARS patients. Emerg Infect Dis 2004; 10:818-824.

14. Hsieh YH, Chen WS, Hsu SB. SARS outbreak, Taiwan, 2003. Emerg Infect Dis 2004; 10:201-206.

15. McDonald LC, Simor AE, Su IJ, et al. SARS in healthcare facilities, Toronto and Taiwan. Emerg Infect Dis 2004; 10:777-781.

16. World Health Organization. Case definitions for surveillance of severe acute respiratory syndrome (SARS). May 1, 2003. Available at: http:// www.who.int/csr/sars/casedefinition/en/. Accessed January 6, 2006.

17. Donnelly CA, Fisher MC, Fraser C, et al. Epidemiological and genetic analysis of severe acute respiratory syndrome. Lancet Infect Dis 2004; 4: 672-683.

18. Leung GM, Hedley AJ, Ho LM, et al. The epidemiology of severe acute respiratory syndrome in the 2003 Hong Kong epidemic: an analysis of all 1755 patients. Ann Intern Med 2004; 141:662-673.

19. Donnelly CA, Ghani AC, Leung GM, et al. Epidemiological determinants of spread of causal agent of SARS in Hong Kong. Lancet 2003; 361: 1761-1766.

20. Karlberg J, Chong DSY, Lai WYY. Do men have a higher case fatality rate of severe acute respiratory syndrome than women do? Am J Epidemiol 2004; 159: 229-231.

21. Center for Disease Control-Taiwan. SARS major timeline. In: Su IJ, ed. Memoir of Severe Acute Respiratory Syndrome Control in Taiwan, 2003. Taipei: CDC-Taiwan; 2003: 67-81.

22. Chen YC, Huang LM, Chan CC, et al. SARS in hospital emergency room. Emerg Infect Dis 2004; 10:782-788.

23. Hsieh YH, Lee JY, Chang HL. SARS epidemiology modeling. Emerg Infect Dis 2004; 10:1165-1167.

24. Moira CY, Yu WC. Outbreak of severe acute respiratory syndrome in Hong Kong Special Administrative Region: case report. BMJ 2003; 326: 850-852.

25. Pang XH, Zhu ZH, Xu FJ, et al. Evaluation of control measures implemented in the severe acute respiratory syndrome outbreak in Beijing, 2003. JAMA 2003; 290: 3215-3221.

26. Zhou G, Yan G. Severe acute respiratory syndrome in Asia. Emerg Infect Dis 2003; 9:1608-1610.

27. Center for Disease Control-Taiwan. Association of the sporadic SARS outbreak in Taiwan. In: Su IJ, ed. Memoir of Severe Acute Respiratory Syndrome Control in Taiwan, 2003. Taipei: CDC-Taiwan; 2003:44.

28. Ho PL, Chau PH, Yip SF, et al. A prediction rule for clinical diagnosis of severe acute respiratory syndrome. Eur Respir J 2005; 26:474-479. 